\title{
Implementation of Multilevel Block Truncation based Face Recognition on Intermediate-4 and Intermediate-9 levels
}

\author{
H.B. Kekre \\ Phd,Senior Professor \\ Computer Engineering \\ Department \\ MPSTME, SVKM's NMIMS \\ (Deemed-to-be University) \\ Mumbai, India
}

\author{
Sudeep Thepade \\ Phd,Associate Professor \\ Computer Engineering \\ Department \\ MPSTME,SVKM's NMIMS \\ (Deemed-to-be University) \\ Mumbai, India
}

\author{
Sanchit Khandelwal, \\ Adnan Azmi, \\ Karan Dhamejani \\ B.Tech Students \\ Computer Engineering \\ Department \\ MPSTME, SVKM's NMIMS \\ (Deemed-to-be University) \\ Mumbai, India
}

\begin{abstract}
The paper presents a performance analysis of Multilevel Block Truncation Coding based Face Recognition on BTCIntermediate- 4 and BTC-Intermediate-9 techniques. In [1], Multilevel Block Truncation Coding was applied on the RGB color space up to four levels for face recognition. Similarly in this paper, Multilevel Block Truncation Coding is implemented on BTC-Intermediate-4 and BTC-Intermediate-9. For experimental analysis, two face databases are used. First one is "Face Database", developed by Dr.Libor Spacek which has 1000 face images and the second one is "Our Own Database" which has 1600 face images. The experimental results showed that Block Truncation Level 4 (BTC-Level 4) gave the best result when applied on whole image as compared to BTCIntermediate-4 and BTC-Intermediate-9 techniques.
\end{abstract}

\section{Keywords}

Face recognition, Block Truncation Coding, RGB, Multilevel BTC.

\section{INTRODUCTION}

The term face recognition refers to identifying and verifying a given face image. Face recognition plays an important role in our day to day lives for identification and authentication purpose. Face recognition can be achieved by comparing the input query face image with the existing face images stored in the database. This identification must be efficient, liable and faster in real time applications.

There are many biometric systems such as finger prints, voice, iris, face and retina. Among these face recognition turns out to be the most effective system since it requires very less human interaction and no explicit cooperation from the users. It is the fastest growing biometric technology.

Some of the applications of face recognition include physical, security and computer access controls, law enforcement [14, 15], criminal list verification, surveillance at various places [17], forensic, authentication at airports [19], etc. Also to capture the image $[23,24,25]$, special equipments are not required.

Many algorithms are used to make face recognition systems effective and efficient. Some of the algorithms include Principle Component Analysis (PCA) [4, 5, 6, 7], Linear Discriminant Analysis (LDA) [8, 9, 10], Independent Component Analysis (ICA) $[11,12,13]$ etc. Also a large number of face detection algorithms are derived from neural network approach, algorithmic approach [26] and some image morphological techniques [20]. However most of the works concentrate on single face detection, with some constrained environments.

In this paper, the performance comparison of Multilevel Block Truncation Coding [1] implementation on whole image i.e. BTC-Full, BTC-Intermediate-4, BTC-Intermediate- 9 has been carried out on two face databases. Results further revealed that the BTC applied on BTC-Full gives better results than that of the implementation of BTC-Intermediate-4 and BTC-Intermediate- 9 at each stage of Multilevel BTC for face recognition.

\section{BLOCK TRUNCATION CODING AND MULTILEVEL BLOCK TRUNCATION CODING}

Block Truncation Coding (BTC) was first developed in 1979 for grayscale image coding [15]. Block truncation coding (BTC) $[13,14,15,16]$ was developed in the early years of digital imaging more than 29 years ago. It is a straightforward and relatively simple image coding technique which demands very less computational complexity. Although it is a simple technique, BTC has played an essential role in the history of digital image coding in the sense that many advanced coding techniques have been developed based on BTC or inspired by the success of BTC.

In the proposed technique, Multilevel Block Truncation Coding, BTC has been implemented using the RGB color space up till four levels [1]. The feature vector size at BTC-Level 1, BTCLevel 2, BTC-Level 3 and BTC-Level 4 is 6, 12, 24 and 48 respectively. In the same way BTC is implemented on Intermediate-4 and Intermediate-9 technique.

\section{BTC-INTERMEDIATE-4 AND BTC- INTERMEDIATE-9}

To calculate the feature vector in this algorithm, Block Truncation Coding has been used. It has been implemented on four levels which are explained below.

\subsection{BTC- Intermediate-4}

\subsubsection{BTC-Intermediate-4 Level 1}

In BTC-Intermediate-4, the given image is partitioned into four non overlapping equal parts. Each part of the image is then considered individually. After this , BTC[1] is applied on each part of the given image .For each part the average intensity value of each of the RGB plane is calculated independently and which helps in dividing the RGB plane into upper and lower clusters respectively. 
Thus we get four feature vectors and each feature vector consists of 6 elements (UR, LR, UG, LG, UB and LB) for an image. Further we calculate the mean value of all the four feature vectors of the four parts of the given image to obtain the feature vector of the entire image $[25,26,27]$.

This is the basic implementation of BTC-Intermediate- 4 method applied on BTC based face recognition level 1 and hence we call it as BTC-Intermediate-4 Level 1.

\subsubsection{BTC-Intermediate-4 Level 2}

In level 2 implementation of BTC based face recognition of BTC-Intermediate-4 we get 12 elements for each feature vector of the four parts of the given image. Thus to obtain the feature vector of the image, the mean value of all the four feature vectors is calculated.

\subsubsection{BTC-Intermediate-4 Level 3 and BTC- \\ Intermediate-4 Level 4}

Similarly in level 3 and level 4 implementation of BTC based face recognition of BTC-Intermediate- 4 we get 24 elements and 48 elements respectively. Thus the mean value is calculated at both the levels in order to obtain the feature vector of the entire image.

\subsection{BTC-Intermediate-9}

The implementation of BTC-Intermediate-9 is same as that of BTC-Intermediate-9, but in this method the image is divided into nine non overlapping equal parts. Similarly to obtain the feature vector of the given image the mean value of all the feature vectors of the nine parts is calculated. The implementation of BTC-Intermediate-9 is also done up to 4 levels.

\section{PROPOSED METHOD}

To calculate the feature vector of each image in the database set and the query image, Multilevel Block Truncation Coding has been implemented for two databases.

At each level of BTC, the feature vector of the query image is compared with the feature vector of each image in the training set. The comparison (Similarity measurement) is done by Mean Square Error (MSE) given by equation 11.

$$
\operatorname{MSE}=\frac{1}{M N} \sum_{y=1}^{M} \sum_{x=1}^{N}[I(x, y)-I(x, y)]^{2}
$$

Where,

I \& I' are two feature vectors of size $\mathrm{M}^{*} \mathrm{~N}$ which are being compared.

False Acceptance Ratio (FAR) and Genuine Acceptance Ratio (GAR) have been used as the performance evaluation parameters to asses the performance of the different BTC levels based face recognition techniques.

\section{IMPLEMENTATION}

\subsection{Platform}

The implementation of the Multilevel BTC is done in MATLAB 2010. It is carried out on a computer using an Intel Core i52410M CPU (2.4 GHz).

\subsection{Database}

The experiments were performed on two face databases.

\section{1) Face Database [18]}

This database is created by Dr Libor consisting of 1000 images (each with 180 pixels by 200 pixels), corresponding to 100 persons in 10 poses each, including both males and females. All the images are captured against a dark or bright homogeneous background, little variation of illumination, different facial expressions and details. The subjects sit at fixed distance from the camera and are asked to speak, whilst a sequence of images is taken. The speech is used to introduce facial expression variation. The images were taken in a single session. The ten poses of Face database are shown in Figure 1.

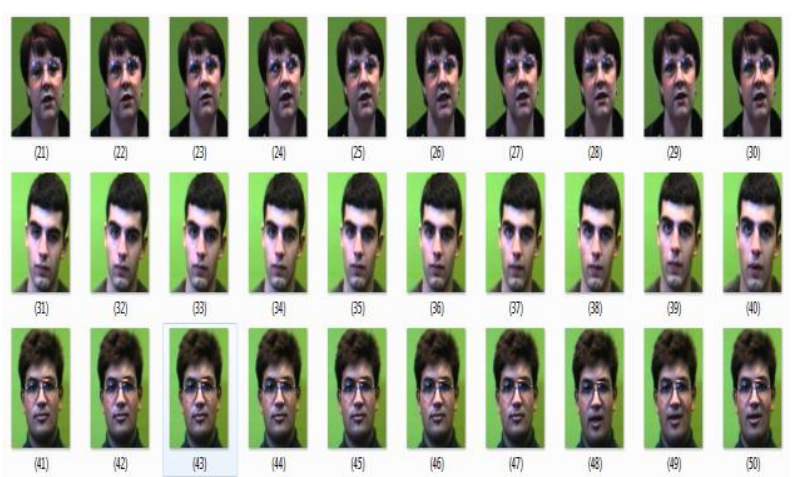

Figure 1: Sample images from Face database

\section{2) Our Own Database [1, 22]}

This database consists of 1600 face images of 160 people (92 males and 68 females).For each person 10 images are taken. The images in the database are captured under numerous illumination settings. The images are taken with a homogenous background with the subjects having different expressions. The images are of variable sizes, unlike the Face database. The ten poses of Our Own Database are shown in Figure 2.

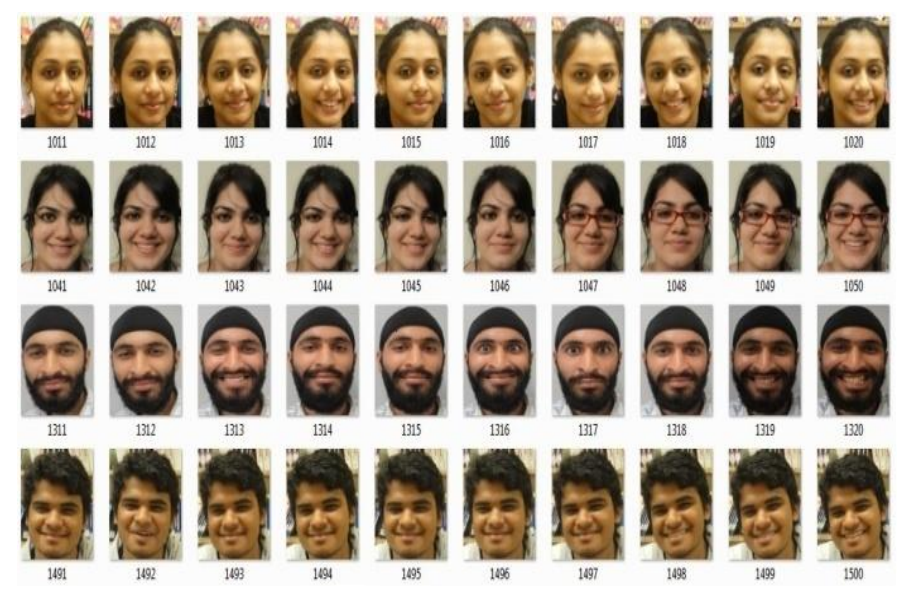

Figure 2: Sample images from Our Own Database

Figure 3 gives the FAR values of the different BTC levels based face recognition techniques tested on face database. Here it can be seen that the FAR values go on decreasing for each succeeding level of BTC. The lesser the FAR value the better is the accuracy. The FAR values obtained by applying the BTC based face recognition techniques on BTC-Full gives the least values at all the four levels as compared to BTC-Intermediate-4 and BTC-Intermediate -9 techniques. The BTC-Full Level 4 gives the best result. 


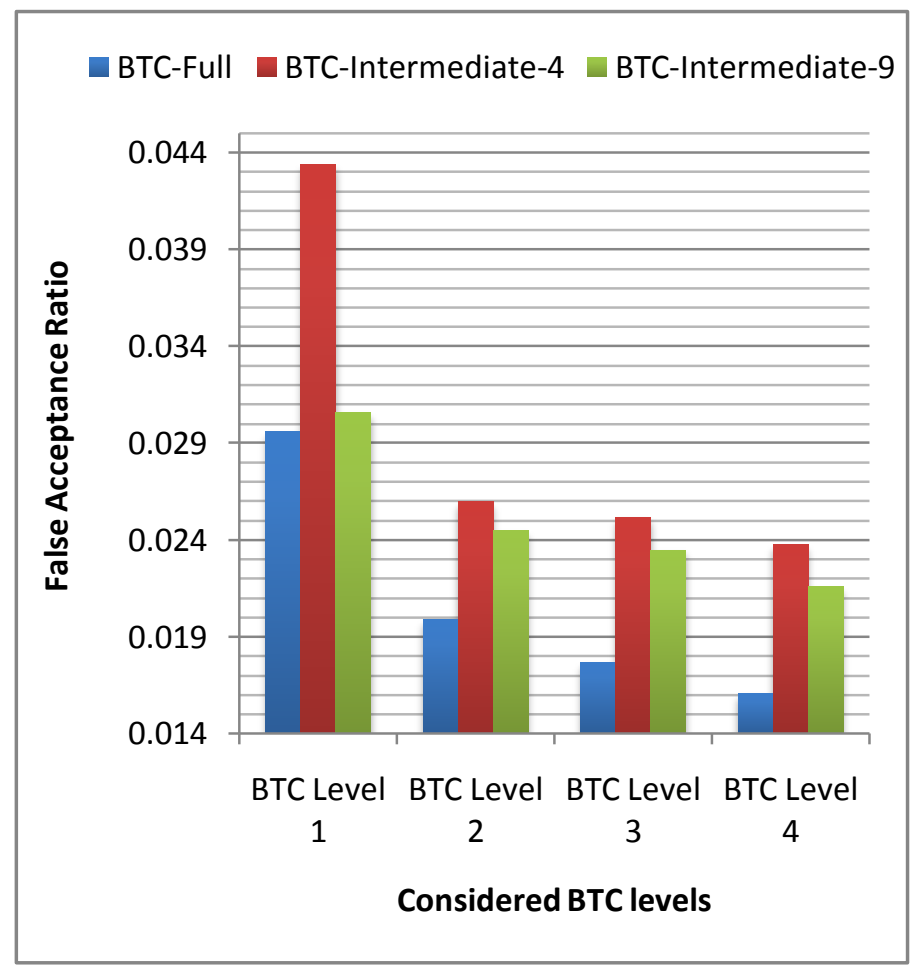

Figure 3: FAR values at different BTC levels BTCFull,BTC-Intermediate-4 and BTC-Intermediate-9 for Face Database.

Table 1. FAR values at different BTC levels BTC-Full, BTCIntermediate-4 and BTC-Intermediate-9 for Face Database

\begin{tabular}{|c|c|c|c|}
\hline Name & \multicolumn{3}{|c|}{ FAR -Face Database } \\
\cline { 2 - 4 } & $\begin{array}{c}\text { BTC- } \\
\text { Full } \\
\text { Intermediate-4 }\end{array}$ & $\begin{array}{c}\text { BTC- } \\
\text { Intermediate-9 }\end{array}$ \\
\hline $\begin{array}{c}\text { BTC- } \\
\text { Level 1 }\end{array}$ & 0.0296 & 0.0434 & 0.0306 \\
\hline $\begin{array}{c}\text { BTC- } \\
\text { Level 2 }\end{array}$ & 0.0199 & 0.026 & 0.0245 \\
\hline $\begin{array}{c}\text { BTC- } \\
\text { Level 3 }\end{array}$ & 0.0177 & 0.0252 & 0.0235 \\
\hline $\begin{array}{c}\text { BTC- } \\
\text { Level 4 }\end{array}$ & 0.0161 & 0.0238 & 0.0216 \\
\hline
\end{tabular}

\section{RESULTS AND DISCUSSIONS}

False Acceptance Rate (FAR) and Genuine Acceptance Rate (GAR) are standard performance evaluation parameters of face recognition system.

The False acceptance rate (FAR) is the measure of the likelihood that the biometric security system will incorrectly accept an access attempt by an unauthorized user. A system's FAR typically is stated as the ratio of the number of false acceptances divided by the number of identification attempts.

$\mathrm{FAR}=($ False Claims Accepted/Total Claims $) \times 100$

The Genuine Acceptance Rate (GAR) is evaluated by subtracting the FAR values from 100.

$$
\text { GAR=100-FAR (in percentage) }
$$

For each color space, 10000 queries (10 images for each of the 1000 people) are fired on face database and 16000 queries (10 images for each of the 1600 people) are fired on Our Own Database. At the end, average FAR and GAR of all queries in respective face databases are considered for performance ranking of BTC levels and of the color spaces.

For optimal performance the FAR values must be less and accordingly the GAR values must be high for each successive levels of BTC.

\subsection{Face Database}

To analyze the performance of proposed algorithm, 10000 queries are tested on the database. The feature vectors of each image for all four BTC levels in RGB color space of BTC-Full, BTC-Intermediate-4 and BTC-Intermediate-9 based face recognition techniques were calculated and then compared with the database. The FAR and GAR values are calculated by employing equations 12 and 13 .

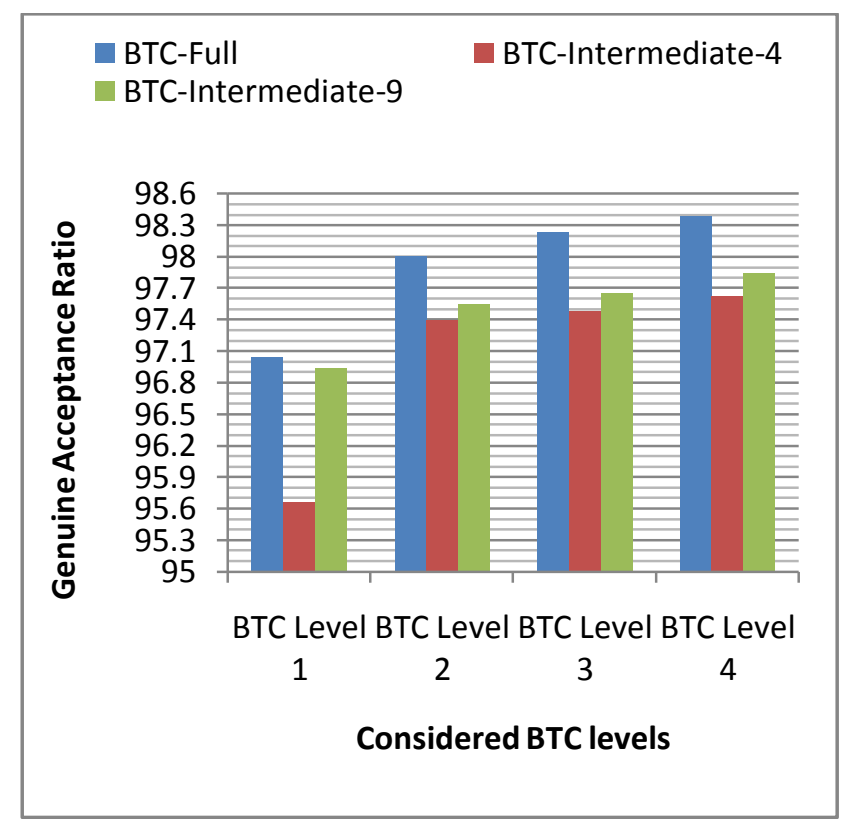

Figure 4: GAR values at different BTC levels BTC-

Full,BTC-Intermediate-4 and BTC-Intermediate-9 for Face Database 
Table 2. GAR values at different BTC levels BTC-Full, BTC Intermediate-4 and BTC-Intermediate-9 for Face Database.

\begin{tabular}{|c|c|c|c|}
\hline \multirow{2}{*}{ Name } & \multicolumn{3}{|c|}{ GAR(Percentage) -Face Database } \\
\cline { 2 - 4 } & $\begin{array}{c}\text { BTC- } \\
\text { Full }\end{array}$ & $\begin{array}{c}\text { BTC- } \\
\text { Intermediate-4 }\end{array}$ & $\begin{array}{c}\text { BTC- } \\
\text { Intermediate-9 }\end{array}$ \\
\hline $\begin{array}{c}\text { BTC-Level } \\
1\end{array}$ & 97.04 & 95.66 & 96.94 \\
\hline $\begin{array}{c}\text { BTC-Level } \\
2\end{array}$ & 98.01 & 97.4 & 97.55 \\
\hline $\begin{array}{c}\text { BTC-Level } \\
3\end{array}$ & 98.23 & 97.48 & 97.65 \\
\hline $\begin{array}{c}\text { BTC-Level } \\
4\end{array}$ & 98.39 & 97.62 & 97.84 \\
\hline
\end{tabular}

Figure 4 gives the GAR values of the different BTC levels based face recognition techniques tested on face database. Here it can be seen that the GAR values go on increasing for each succeeding level of BTC. The greater the GAR value the better is the accuracy .The GAR values obtained by applying the BTC based face recognition techniques on BTC-Full gives the highest GAR values at all the four levels as compared to BTCIntermediate-4 and BTC-Intermediate -9 techniques .The BTCFull level-4 gives the best result.

For optimal performance the FAR values must be less and accordingly the GAR values must be high for each successive levels of BTC. Thus, the performance of whole image implementation for BTC levels based face recognition techniques is superior to the performance of BTC-Intermediate-4 and BTC-Intermediate-9 implementation for BTC based face recognition techniques.

\subsection{Our Own Database}

In all 16000 queries were tested on the database for analyzing the performance of the proposed BTC levels based face recognition algorithm for BTC-Full, BTC-Intermediate-4 and BTC-Intermediate-9 based face recognition techniques. Similarly the feature vectors of each image for all four BTC levels in RGB color space of BTC-Full, BTC-Intermediate-4 and BTC-Intermediate- 9 based face recognition techniques were calculated and then compared with the database. The FAR and GAR values are calculated by employing equations 12 and 13 .
- BTC-Full $\square$ BTC-Intermediate-4 $=$ BTC-Intermediate-9

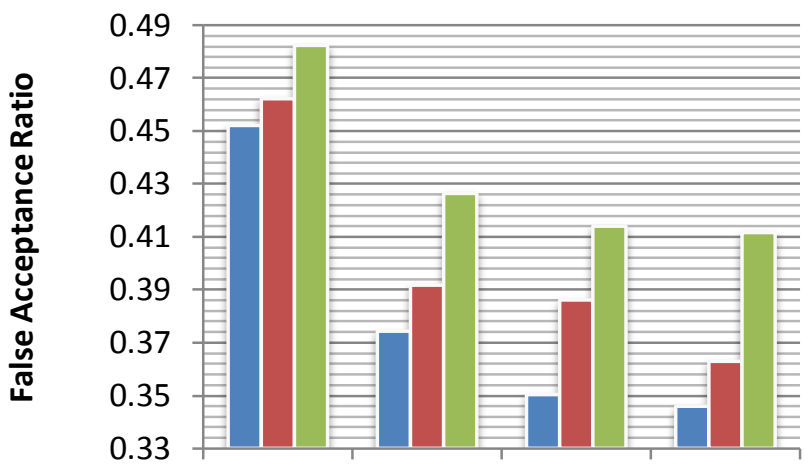

BTC Level BTC Level BTC Level BTC Level 1

$$
2
$$

3

4

\section{Considered BTC levels}

Figure 5: FAR values at different BTC levels BTCFull,BTC-Intermediate-4 and BTC-Intermediate-9 for Our Own Database.

Table 3. FAR values at different BTC levels BTC-Full,BTCIntermediate-4 and BTC-Intermediate-9 for Our Own Database.

\begin{tabular}{|c|c|c|c|}
\hline \multirow{2}{*}{ Name } & \multicolumn{3}{|c|}{ FAR -Face Database } \\
\cline { 2 - 4 } & $\begin{array}{c}\text { BTC- } \\
\text { Full }\end{array}$ & $\begin{array}{c}\text { BTC- } \\
\text { Intermediate-4 }\end{array}$ & $\begin{array}{c}\text { BTC- } \\
\text { Intermediate-9 }\end{array}$ \\
\hline $\begin{array}{c}\text { BTC- } \\
\text { Level 1 }\end{array}$ & 0.4521 & 0.4622 & 0.4824 \\
\hline $\begin{array}{c}\text { BTC- } \\
\text { Level 2 }\end{array}$ & 0.3743 & 0.3917 & 0.4264 \\
\hline $\begin{array}{c}\text { BTC- } \\
\text { Level 3 }\end{array}$ & 0.3503 & 0.3861 & 0.4116 \\
\hline $\begin{array}{c}\text { BTC- } \\
\text { Level 4 }\end{array}$ & 0.3459 & 0.3629 & 0.414 \\
\hline
\end{tabular}

Figure 5 gives the FAR values of the different BTC levels based face recognition techniques tested on Our Own Database .Here it can be seen that the FAR values go on decreasing for each succeeding level of BTC. Thus, when BTC based face recognition techniques is applied on Our Own Database, it gives a result similar to the Face Database.The BTC-Full level 4 gives the best result in comparison with BTC-Intermediate-4 and BTC-Intermediate- 9 based face recognition techniques. 
BTC-Full $\square$ BTC-Intermediate-4 BTC-Intermediate-9

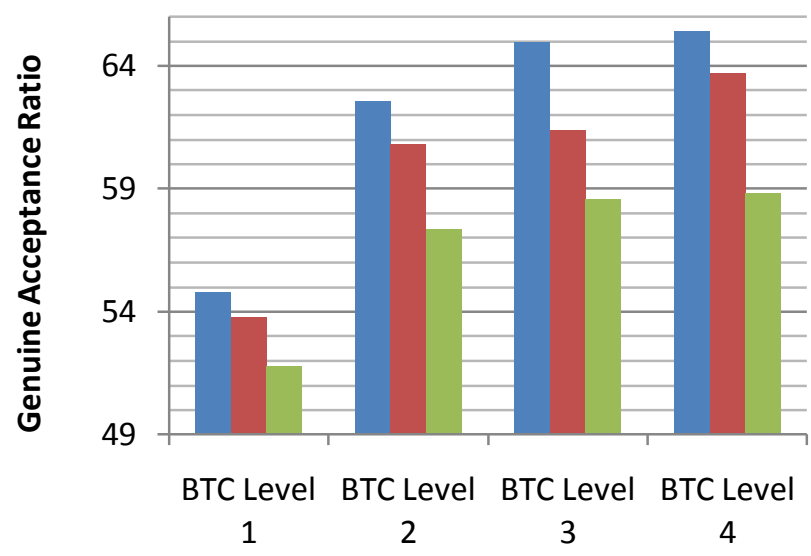

Considered BTC levels

Figure 6: GAR values at different BTC levels BTC-Full, BTC-Intermediate-4 and BTC-Intermediate-9 for Our Own Database

Table 4. GAR values at different BTC levels BTC-Full, BTCIntermediate-4 and BTC-Intermediate-9 for Our Own Database.

\begin{tabular}{|c|c|c|c|}
\hline \multirow{2}{*}{ Name } & \multicolumn{3}{|c|}{ GAR(Percentage)-Our Own Database } \\
& $\begin{array}{c}\text { BTC- } \\
\text { Full }\end{array}$ & $\begin{array}{c}\text { BTC- } \\
\text { Intermediate-4 }\end{array}$ & $\begin{array}{c}\text { BTC- } \\
\text { Intermediate-9 }\end{array}$ \\
\hline BTC-Level 1 & 54.79 & 53.78 & 51.76 \\
\hline BTC-Level 2 & 62.57 & 60.83 & 57.36 \\
\hline BTC-Level 3 & 64.97 & 61.39 & 58.6 \\
\hline BTC-Level 4 & 65.41 & 63.71 & 58.84 \\
& & & \\
\hline
\end{tabular}

Figure 6 gives the GAR values of the different BTC levels based face recognition techniques tested on Our Own Database .Here it can be seen that the GAR values go on increasing for each succeeding level of BTC. The GAR values obtained by applying the BTC based face recognition techniques on BTC-Full gives the highest GAR values at all the four levels as compared to BTC-Intermediate-4 and BTC-Intermediate -9 based face recognition techniques. The BTC-Full level-4 gives the best result while BTC-Intermediate-9 gives the worst.

As seen from the performance of both the databases it can be concluded BTC-FULL based face recognition technique is superior and much better than that of the BTC-Intermediate-4 and BTC-Intermediate-9 based face recognition techniques.

\section{CONCLUSION}

It can be concluded that partitioning the image further into 4 parts (BTC-Interemediate-4) and 9 parts (BTC-Intermediate-9) and applying Block Truncation Coding on them serves no good and hence should be avoided. The implementation of BTC based face recognition techniques on the whole image (BTC-Full) gives better results as compared to BTC-Intermediate- 4 and BTC-Intermediate-9 based face recognition techniques.

\section{REFERENCES}

[1] H.B.Kekre, Sudeep D. Thepade, Sanchit Khandelwal, Karan Dhamejani, Adnan Azmi, "Face Recognition using Multilevel Block Truncation Coding" International Journal of Computer Applications (IJCA) December 2011 Edition.

[2] Dr. H. B. Kekre, Sudeep Thepade, Karan Dhamejani, Adnan Azmi, Sanchit Khandelwal, "Improved Face Recognition with Multilevel BTC using Kekre's LUV Color Space", IJACSA

[3] Dr. H. B. Kekre, Sudeep Thepade, Karan Dhamejani, Adnan Azmi, Sanchit Khandelwal, "Performance Comparison of Assorted Color Spaces for Multilevel Block Truncation Coding based Face Recogniton”, IJCSIS

[4] Xiujuan Li, Jie Ma and Shutao Li 2007. A novel faces recognition method based on Principal Component Analysis and Kernel Partial Least. IEEE International Conference on Robotics and Biometrics, 2007. ROBIO 2007

Shermin J "Illumination invariant face recognition using Discrete Cosine Transform and Principal Component Analysis" 2011 International Conference on Emerging Trends in Electrical and Computer Technology (ICETECT).

Zhao Lihong, Guo Zikui "Face Recognition Method Based on Adaptively Weighted Block-Two Dimensional Principal Component Analysis"; 2011 Third International Conference on Computational Intelligence, Communication Systems and Networks (CICSyN)

Gomathi, E, Baskaran, K. "Recognition of Faces Using Improved Principal Component Analysis"; 2010 Second International Conference on Machine Learning and Computing (ICMLC)

Haitao Zhao, Pong Chi Yuen" Incremental Linear Discriminant Analysis for Face Recognition", IEEE Transactions on Systems, Man, and Cybernetics, Part B: Cybernetics

[9] Tae-Kyun Kim; Kittler, J. "Locally linear discriminant analysis for multimodally distributed classes for face recognition with a single model image" IEEE Transactions on Pattern Analysis and Machine Intelligence, March 2005

[10] James, E.A.K., Annadurai, S. "Implementation of incremental linear discriminant analysis using singular value decomposition for face recognition". First International Conference on Advanced Computing, 2009. ICAC 2009

[11] Zhao Lihong, Wang Ye, Teng Hongfeng; "Face recognition based on independent component analysis", 2011 Chinese Control and Decision Conference (CCDC)

[12] Yunxia Li, Changyuan Fan; "Face Recognition by Non negative Independent Component Analysis" Fifth 
International Conference on Natural Computation, 2009. ICNC'09'.

[13] Yanchuan Huang, Mingchu Li, Chuang Lin and Linlin Tian. "Gabor-Based Kernel Independent Component Analysis on Intelligent Information Hiding and Multimedia Signal Processing (IIH-MSP).

[14] H.B.Kekre, Sudeep D. Thepade, Varun Lodha, Pooja Luthra, Ajoy Joseph, Chitrangada Nemani "Augmentation of Block Truncation Coding based Image Retrieval by using Even and Odd Images with Sundry Color Spaces" ,Int. Journal on Computer Science and Engg. Vol. 02, No. 08, 2010, 2535-2544

[15] H.B.Kekre, Sudeep D. Thepade, Shrikant P. Sanas, "Improved CBIR using Multileveled Block Truncation Coding" ,International Journal on Computer Science and Engineering Vol. 02, No. 08, 2010, 2535-2544

[16] H.B.Kekre, Sudeep D. Thepade, "Boosting Block Truncation Coding using Kekre's LUV Color Space for Image Retrieval", WASET International Journal of Electrical, Computer and System Engineering (IJECSE), Volume 2, Number 3, pp. 172-180, Summer 2008.

[17] H.B.Kekre, Sudeep D. Thepade, "Image Retrieval using Augmented Block Truncation Coding Techniques", ACM International Conference on Advances in Computing, Communication and Control (ICAC3- 2009), pp. 384-390, 23-24 Jan 2009, Fr. Conceicao Rodrigous College of Engg., Mumbai

[18] Developed by Dr. Libor Spacek. Available Online at: http://cswww.essex.ac.uk/mv/otherprojects.html

[19] Mark D. Fairchild, "Color Appearance Models", 2nd Edition, Wiley-IS\&T, Chichester, UK, 2005. ISBN 0-47001216-1

[20] Rafael C. Gonzalez and Richard Eugene Woods "Digital Image Processing", 3rd edition, Prentice Hall, Upper Saddle River, NJ, 2008. ISBN 0-13-168728-X. pp. 407413.S

[21] Dr.H.B.Kekre, Sudeep D. Thepade and Shrikant P. Sanas, "Improved CBIR using Multileveled Block Truncation Coding”, (IJCSE) International Journal on Computer Science and Engineering Vol. 02, No. 07, 2010, 2471-2476

[22] Dr. H.B.Kekre, Sudeep D. Thepade and Akshay Maloo, "Face Recognition using Texture Feartures Extracted from Walshlet Pyramid ", Int. J. on Recent Trends in Engineering \& Technology, Vol. 05, No. 01, Mar 2011.

[23] Koji kotani, Chen Qiu and Tadahiro Ohmi, "Face Recognition Using Vector Quantization Histogram Method". International Conference on Image Processing,Volume 2, pp.105-108,2002.

[24] Shang-Hung Lin, "An Introduction to Face Recognition Technology", Informing Science Special Issue on Multimedia Informing Technologies- Part 2, Volume 3 No.1, 2000.

[25] H. B. Kekre, Sudeep Thepade, Akshay Maloo, "Eigenvectors of Covariance Matrix using Row Mean and Column Mean Sequences for Face Recognition", CSCInternational Journal of Biometrics and Bioinformatics (IJBB), Volume (4): Issue (2), pp. 42-50, May 2010.
[26] H. C. Vijaya Lakshmi, D. Patil Kulakarni "Segmentation algorithm for multiple face detection in color images with skin tone regions using color spaces and edge detection techniques," International journal of computer theory and engineering 1793-8201,2010.

\section{AUTHOR'S PROFILE}

Dr. H. B. Kekre has received B.E. (Hons.) in Telecomm. Engineering. from Jabalpur University in 1958, M.Tech (Industrial Electronics) from IIT Bombay in 1960, M.S.Engg. (Electrical Engg.) from University of Ottawa in 1965 and Ph.D. (System Identification) from IIT Bombay in $1970 \mathrm{He}$ has worked as Faculty of Electrical Engg. and then HOD Computer Science and Engg. at IIT Bombay. For 13 years he was working as a professor and head in the Department of Computer Engg. at Thadomal Shahani Engineering. College, Mumbai. Now he is Senior Professor at MPSTME, SVKM" s NMIMS University. He has guided $17 \mathrm{Ph} . \mathrm{Ds}$, more than 100 M.E./M.Tech and several B.E./B.Tech projects. His areas of interest are Digital Signal processing, Image Processing and Computer Networking. $\mathrm{He}$ has more than 350 papers in National / International Conferences and Journals to his credit. He was Senior Member of IEEE. Presently He is Fellow of IETE and Life Member of ISTE Recently ten students working under his guidance have received best paper awards and two have been conferred Ph.D. degree of SVKM" sNMIMS University. Currently 10 research scholars are pursuing $\mathrm{Ph} . \mathrm{D}$. program under his guidance.

Dr. Sudeep D. Thepade has Received B.E.(Computer) degree from North Maharashtra University with Distinction in 2003, M.E. in Computer Engineering from University of Mumbai in 2008 with Distinction, Ph.D. from SVKM" s NMIMS (Deemed to be University) in July 2011, Mumbai. He has more than 08 years of experience in teaching and industry. He was Lecturer in Dept. of Information Technology at Thadomal Shahani Engineering College, Bandra(W), Mumbai for nearly 04 years. Currently working as Associate Professor in Computer Engineering at Mukesh Patel School of Technology Management and Engineering, SVKM ${ }^{\text {ec }}$ s NMIMS (Deemed to be University), Vile Parle (W), Mumbai, INDIA. He is member of International Advisory Committee for many International Conferences, acting as reviewer for many referred international journals/transactionsincluding IEEE and IET. His areas of interest are Image Processing and Biometric Identification. He has guided five M.Tech. projects and several B.Tech projects. $\mathrm{He}$ has more than 130 papers in National/International Conferences/Journals to his credit with a Best Paper Award at International Conference SSPCCIN-2008, Second Best Paper Award at ThinkQuest-2009, Second Best Research Project Award at Manshodhan 2010, Best Paper Award for paper published in June 2011 issue of International Journal IJCSIS (USA), Editor" s Choice Awards for papers published in International Journal IJCA (USA) in 2010 and 2011.

Sanchit Khandelwal is currently pursuing B.Tech. (CE) from MPSTME, NMIMS University, Mumbai. His areas of interest are Image Processing and Computer Networks and security. $\mathrm{He}$ has 3 paper in an international journal to his credit.

Karan Dhamejani is currently pursuing B.Tech. (CE) from MPSTME, NMIMS University, Mumbai. His areas of interest are Image Processing, Computer Networks and UNIX programming. He has 4 papers in an international journal to his credit.

Adnan Azmi is currently pursuing B.Tech. (CE) from MPSTME, NMIMS University, Mumbai. His areas of interest are Image Processing and Computer Networks. He has 3 papers in an international journal to his credit 